\title{
El empleo de herramientas de la Web 2.0 para el desarrollo de estrategias cognitivas: un estudio comparativo
}

\author{
URTZA GARAY \\ $U P V-E H U$ \\ CARMEN LUJÁN \\ Universidad de Las Palmas de Gran Canaria \\ AinTZANE ETXEBARRIA \\ $U P V-E H U$
}

Recibido: 1 de noviembre 2011 / Aceptado: 13 febrero de 2013

ISSN: $1697-7467$

\begin{abstract}
RESUMEN: La introducción de Internet en las aulas universitarias abre un nuevo abanico de posibilidades. Los instrumentos que aporta la Web 2.0 contribuyen al desarrollo de las estrategias cognitivas, esenciales para el aprendizaje autónomo de los alumnos. Pero, ¿cuál es el uso real que realiza el alumnado universitario actual de estas herramientas para desarrollar de forma consciente sus estrategias cognitivas? En este trabajo contestamos a esta pregunta, además de comparar los resultados obtenidos entre alumnos del mismo nivel académico pero con gran distancia geográfica, como son los de la Universidad del País Vasco (UPV-EHU) y de la Universidad de Las Palmas de Gran Canaria.

Palabras clave: Web 2.0, estrategias cognitivas, enseñanza-aprendizaje universitario, autonomía.
\end{abstract}

\section{Using Web 2.0 Tools for Developing Cognitive Strategies: a Comparative Study}

\begin{abstract}
The use of the internet in University classrooms opens a new range of possibilities. Web 2.0 provides a variety of tools that contribute to the development of cognitive strategies, which are essential for students' autonomous learning. However, how do current university students actually use these tools to consciously develop their cognitive strategies? In this paper, we answer this question, in addition to comparing the results found amongst students of the same academic level in two universities which are geographically distant from each other; namely the University of the Basque country and the University of Las Palmas de Gran Canaria.

Keywords: WEB 2.0, cognitive strategies, university teaching-learning, autonomous.
\end{abstract}

\section{Las estrategias Cognitivas}

Las estrategias de aprendizaje son un grupo de acciones que nos facilitan el camino en el proceso de aprendizaje No son tácticas, sino habilidades específicas o instrumentos que se emplean dentro del marco de un plan general, llamado estrategia, en el proceso de aprendizaje 
(Gallego, 1997: 23). A lo largo de los últimos años, estas estrategias de aprendizaje se han clasificado de muchas maneras, en el año $2000 \mathrm{P}$. Cyr publica un libro en el que recoge algunas de las clasificaciones: la de Rubin (Rubin: 1981) en la que se distinguen dos tipos de estrategias llamadas directas y transversales; la de Oxford (Oxford: 1990) que sigue el camino del anterior, pero concreta mucho más; y por último, según Cyr la más conocida y valorada, es la de O'Malley y Chamot (O'Malley y Chamot: 1990) en la que se distinguen tres grupos de estrategias que tal y como se citarán más adelante son las cognitivas, metacognitivas y socio-afectivas. En este artículo nos centraremos en las estrategias cognitivas que han sido definidas de diversas formas. F. Alonso apunta que son recursos cognitivos, afectivos o psicomotores los que el sujeto lleva a cabo en los procesos de cumplimiento de objetivos de razonamiento, memoria o aprendizaje (Alonso, 1993: 121).

En 1997, Skehan, siguiendo el camino de Rubin, menciona que son estrategias directas esenciales para el proceso del aprendizaje:

Cognitive strategies are concerned with the direct activities that are engaged in to promote learning. They would include such things as memorization techniques, inferencing, and so on. They represent what learners actually do to process the material that needs to be learned, and would correspond to Rubin's (1981: 265) direct learning strategies. (Skehan, 1997:73).

De todas formas, algunos autores sostienen que este tipo de estrategias están estrechamente relacionadas con la metacognición, que según Flavell (Flavell: 1993) alude a reacciones o reflexiones relativamente espontáneas que ocurren antes, durante y después del proceso cognitivo (Crespo: 2004). De ese modo, la metacognición estaría relacionada con el conocimiento de las estrategias cognitivas que se aplican en el proceso de aprendizaje. Alonso señala que las estrategias metacognitivas son para las estrategias cognitivas lo que éstas son para las destrezas o habilidades, es decir, que si las estrategias organizan el uso de las destrezas de forma secuenciada para obtener un fin cognitivo, las estrategias metacognitivas en base a autoinstrucciones mantienen las condiciones óptimas para la aplicación de las estrategias en el transcurso de los procesos de aprendizaje, de modo que se consiga el objetivo de las tareas. (Alonso: 1993: 131). A pesar de que las estrategias de aprendizaje cognitivas y metacognitivas son complementarias, en este trabajo solamente nos hemos centrado en las cognitivas que han sido estudiadas por numerosos investigadores (Oxford, 1990; Politzer \& McGroarty, 1985; O’Malley \& Chamot, 1990; Skehan, 1997; Rubin, 1981; Villanueva, 1997). Así, trabajaremos sobre las clasificaciones de Oxford y O’Malley \& Chamot por ser las más conocidas.

Oxford, en 1990, clasifica las estrategias cognitivas dentro del grupo de las directas junto con las mnemónicas y compensatorias. Las mnemónicas son las que ayudan al estudiante a almacenar y recuperar información; por ejemplo, establecer relaciones entre imágenes y sonidos, unir palabras por su significado, repasar de vez en cuando, valerse de técnicas mecánicas. Las cognitivas son estrategias para practicar, recibir y enviar información, razonar y analizar estructuras para su construcción en diferentes contextos. Por último están las estrategias compensatorias, que pueden ser tanto linguísticas como extra-lingüísticas y ayudan a completar la información ante las dificultades que pueda tener el estudiante (paráfrasis, gestos, utilización de la lengua materna, etc.). En las siguientes líneas se muestran las denominadas estrategias directas de Oxford, dentro de las que se encuentran las cognitivas: 


\begin{tabular}{|l|l|l|}
\hline \multicolumn{2}{|c|}{ ESTRATEGIAS DIRECTAS } \\
\hline \multicolumn{1}{|c|}{ De memoria } & \multicolumn{1}{|c|}{ Cognitivas } & \multicolumn{1}{c|}{ Estrategias de compensación } \\
\hline - Creación de enlaces & $\cdot$ Práctica & $\cdot$ Adivinar de manera inteligente \\
mentales & $\cdot$ Recibir y enviar mensajes & $\begin{array}{l}\text { Superar limitaciones en la } \\
\text { expresión oral y la escrita }\end{array}$ \\
$\cdot$ Aplicación de imágenes y & estratégicos & \\
sonidos & $\cdot$ Analizar y razonar & \\
$\cdot$ Revisión & Crear estructuras de entrada y & \\
Empleo de la acción & de salida & \\
& & \\
\hline
\end{tabular}

Figura 1: Tabla resumen de estrategias directas (adaptación de Oxford, 1990).

O'Malley y Chamot ese mismo año, tal y como se ha mencionado anteriormente, diferencian tres grupos de estrategias, una de ellos corresponde a las estrategias cognitivas, que las definen como un conjunto de técnicas que sirven para llevar a cabo tareas específicas, e identifican once tipos de estrategias: repetition, resourcing, grouping, note taking, deduction/ induction, substitution, elaboration, summarization, translation, transfer, inferencing.

Por último, este tipo de estrategias de aprendizaje tienen la ventaja de ser muy específicas y fáciles de observar directa e indirectamente (Cyr, 2000: 48). Aunque se ha cuestionado mucho si se deberían enseñar de una manera explícita, creemos que su aplicación de manera integrada en la enseñanza de materias a través de diversas herramientas ayuda en el aprendizaje autónomo y significativo del alumno.

\section{La APORTACión de la Web 2.0 a las estrategias de APRENDizaje}

La introducción de las herramientas que engloba la Web 2.0 en la educación lingüística ha propiciado un cambio de punto de vista. Ha provocado un cambio de actitud tanto hacia el aprendizaje, pero mucho más hacia la enseñanza; ya que son herramientas cuyo uso posibilita dar realidad a esa necesidad de que el alumno de lenguas se convierta en protagonista real de su proceso de aprendizaje, mientras que el profesor será el guía en la enseñanza.

Pero para garantizar el buen desarrollo y el éxito de estas herramientas que la Web propone lo cierto es que se consolida como pilar esencial el cómo se utilizan y el punto de vista con el que se realiza su uso en la educación lingüística superior, en este caso. Por ello, es necesario conocer el procedimiento de uso de la herramienta, esto es, cómo se utiliza. Pero más importante es planificar de forma concienciada su uso para la consecución de objetivos planteados para el proceso de enseñanza-aprendizaje. Y es en este punto donde toman especial relevancia las estrategias de aprendizaje lingüístico. Las herramientas de la Web 2.0 proporcionan un lugar virtual, un contexto de enseñanza-aprendizaje real, donde alumnos y profesores pueden desarrollar de forma planificada dichas estrategias.

En este trabajo nos hemos centrado en el uso de estrategias de aprendizaje cognitivas, estrategias cuyas características pueden vincularse de forma directa con las ventajas que proporciona la utilización planificada y concienzuda de las herramientas de la Web 2.0 dentro del desarrollo del proceso de enseñanza-aprendizaje de una segunda lengua. 
Así tal y como podemos observar en la tabla siguiente, las estrategias sociales se caracterizan por ser actividades para practicar lo que se está aprendiendo, para recopilar y para gestionar la información. La Web 2.0 proporciona diversas herramientas para poder llevar a cabo estas actividades como son, entre otras, los wikis, blogs, foros, podcasts para practicar y eportfolios, PLEs y de nuevo blogs para su gestión y recopilación; lo que contribuye a llevar adelante un proceso de enseñanza y aprendizaje actual basado en estrategias de aprendizaje, tal y como se puede observar en el siguiente esquema:

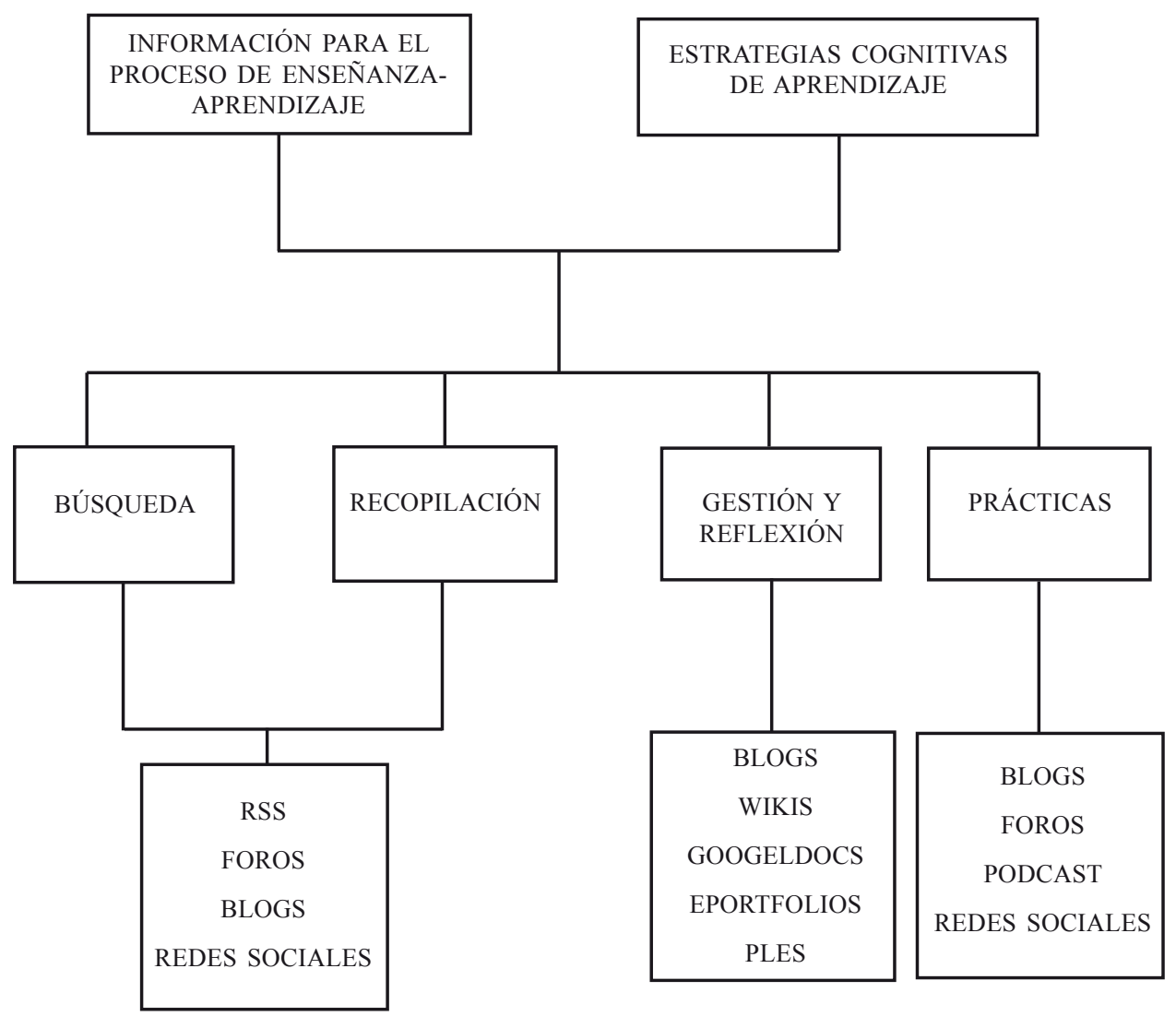

Figura 2: Estrategias cognitivas y herramientas Web 2.0 para la gestión de la información.

Por lo tanto, las herramientas que la web aporta facilitan el desarrollo de la enseñanza y el aprendizaje basado en estrategias, lo que no significa que el hecho de utilizar estas herramientas conlleve el desarrollo de dichas estrategias. Ya que no podemos olvidar que lo que internet nos propone como novedoso no deja de ser una mera herramienta de trabajo, y que su buen funcionamiento estará supeditado a la utilización que se haga de ello y al punto de vista y el objetivo con que se realice. 


\section{ObJetivos}

El presente trabajo tiene una doble finalidad.

En primer lugar, determinar el uso real que el alumnado universitario encuestado hace de las herramientas que aporta la Web 2.0 para desarrollar sus estrategias cognitivas de forma consciente.

En segunda lugar, establecer una comparativa entre los resultados obtenidos entre los estudiantes de dos universidades públicas españolas: Universidad del País Vasco y Universidad de Las Palmas de Gran Canaria.

\section{MÉTOdo Y Muestra}

Con el objetivo de recoger y medir las aportaciones del alumnado se realizó una encuesta implementada anónimamente en dos universidades españolas: Universidad de Las Palmas de Gran Canaria y Universidad del País Vasco (UPV-EHU). La encuesta fue realizada por varios profesores de dichas universidades sobre un universo de 147 alumnos. Los alumnos pertenecían a $2^{\circ}$ curso de Magisterio, especialidad Diplomatura en Educación Infantil, y de $2^{\circ}$ curso de Filología Inglesa y de $1^{\circ}$ del grado en Lenguas Modernas (titulación que viene a sustituir la licenciatura de filología inglesa con el nuevo sistema EEES). Las edades de los informantes oscilaban entre 18 y 40 años, aunque la mayoría pertenecía a la franja entre 18 y 20 años. La siguiente tabla ofrece un desglose detallado de las edades de los participantes en este estudio.

Tabla 1. Edad de los sujetos.

\begin{tabular}{llll} 
& Porcentaje & Frecuencia & N \\
\hline $18-20$ años & 49 & 72 & \\
$21-30$ años & 43,5 & 64 & \\
$31-40$ años & 7,5 & 11 & 147 \\
Número total & & &
\end{tabular}

Otro de los datos recogidos como variable posterior fue el género de los encuestados de los que la siguiente tabla aporta un desglose.

Tabla 2. Género de los sujetos.

\begin{tabular}{llll} 
& Porcentaje & Frecuencia & N \\
\hline Hombres & 25,3 & 37 & \\
Mujeres & 74,7 & 109 & 146 \\
Número total & & & \\
\hline
\end{tabular}


Como se puede observar en la anterior tabla, existe un mayor porcentaje de mujeres $(74,7)$ que de hombres $(25,3)$, ya que el estudio se realizó entre titulaciones que tradicionalmente han contado con un mayor número de alumnado femenino que masculino. Por tanto, en este trabajo no se hará un análisis comparativo entre ambos sexos ya que los porcentajes entre ambos son bastante dispares.

Como ya se ha mencionado, el presente estudio se realizó entre dos universidades: la Universidad del País Vasco y la Universidad de Las Palmas de Gran Canaria, con el objetivo final de establecer si existían diferencias en torno al uso de la Web 2.0 por parte del alumnado de las dos universidades y las dos titulaciones: Magisterio (Universidad del País Vasco) y Filología Inglesa/grado en Lenguas Modernas (Universidad de Las Palmas de Gran Canaria). Ambas universidades, a pesar de ser españolas y de tener carácter público, se encuentran bastante distantes geográficamente. La siguiente tabla desglosa el alumnado perteneciente a cada universidad.

Tabla 3. Universidades de los sujetos.

\begin{tabular}{llll} 
& Porcentaje & Frecuencia & N \\
\hline País Vasco & 49 & 72 & \\
Las Palmas de Gran Canaria & 51 & 75 & 147 \\
Número total & & & \\
\hline
\end{tabular}

Además, se elaboró una encuesta teniendo en cuenta la relación existente entre distintas estrategias cognitivas básicas para la enseñanza y el aprendizaje de segundas lenguas y las ventajas, o características, que aporta la utilización de herramientas que ofrece la Web 2.0 para dicho fin.

Pero la elaboración de las preguntas unidas a la vida académica del alumnado se basó en la unión de las estrategias y su desarrollo mediante la utilización de herramientas Web 2.0, tal y como se puede observar en la siguiente tabla:

\begin{tabular}{|c|l|l|}
\hline Estrategia cognitiva & \multicolumn{1}{|c|}{ Herramienta Web 2.0 } & \multicolumn{1}{c|}{ Ítem } \\
\hline \multirow{2}{*}{$\begin{array}{c}\text { BÚSQUEDA Y } \\
\text { RECOPILACIÓN (completar } \\
\text { información) }\end{array}$} & Foros & $\begin{array}{l}\text {-Para recopilar información } \\
\text { utilizo Foros. }\end{array}$ \\
\cline { 2 - 3 } & Blogs & $\begin{array}{l}\text {-Para recopilar información } \\
\text { consulto Blogs. }\end{array}$ \\
\cline { 2 - 3 } & & $\begin{array}{l}\text {-Para completar información } \\
\text { que recibo en clase utilizo } \\
\text { marcadores sociales. }\end{array}$ \\
\hline \multirow{2}{*}{$\begin{array}{c}\text { GESTIONAR Y Y } \\
\text { REFEXIONAR }\end{array}$} & Blogs / Foros & $\begin{array}{l}\text {-Reflexionar en Blogs o Foros } \\
\text { para poner en práctica lo que } \\
\text { he aprendido. }\end{array}$ \\
\cline { 2 - 3 } & Wikis / Google Docs & $\begin{array}{l}\text {-Para elaborar trabajos en } \\
\text { grupo con mis compañeros } \\
\text { utilizo wikis o Google docs. }\end{array}$ \\
& & \\
& &
\end{tabular}




\begin{tabular}{|l|l|l|}
\hline \multirow{2}{*}{ PRACTICAR } & Podcast & $\begin{array}{l}\text {-Utilizar Podcast para practicar } \\
\text { de forma oral lo que aprendo. }\end{array}$ \\
\cline { 2 - 3 } & Blogs / Foros & $\begin{array}{l}\text { - Reflexionar en Blogs o Foros } \\
\text { para poner en práctica lo que } \\
\text { he aprendido. }\end{array}$ \\
\cline { 2 - 3 } & Redes Sociales & $\begin{array}{l}\text {-Practicar mediante la } \\
\text { reflexión lo que he aprendido } \\
\text { en Redes Sociales tales como } \\
\text { Facebook, Tuenti o Twitter. }\end{array}$ \\
\hline
\end{tabular}

Figura 3: Relación entre estrategias cognitivas, herramientas Web 2.0 e items.

Finalmente, las posibles respuestas entre las que podía elegir el alumno-participante para dar su respuesta conformaban una escala de Likert que constaba de cuatro puntos, tal y como se puede observar a continuación:
a. Totalmente de acuerdo
b. De acuerdo
c. En desacuerdo
d. Totalmente en desacuerdo

Dada la amplitud de este estudio, en el presente artículo nos centraremos en el estudio de dos aspectos distintos: 1 . un análisis general de los resultados obtenidos concentrándonos en los tres bloques de uso de la Web 2.0 con fines académicos. 2. un estudio comparativo de los resultados obtenidos entre los informantes de las dos universidades objeto de este estudio.

\section{Estudio de CAMPo y Resultados}

En primer lugar, realizaremos un análisis de los resultados en términos generales, sin distinción entre variables, sino con el empleo de la escala de Likert descrita en la sección anterior.

\subsection{Empleo de los recursos de la web 2.0}

El primer bloque que se analizó atendía al empleo de distintos recursos de la Web 2.0 con el objeto de buscar y recopilar información de carácter académico. 


\subsubsection{Bloque A: Búsqueda y recopilación}

Tabla 1. Uso de foros para recopilar información académica

\begin{tabular}{|c|c|c|c|}
\hline Total & Frecuencia & Porcentaje & \\
\hline Total desacuerdo & 29 & 19,9 & \\
\hline Desacuerdo & 70 & 47,9 & \\
\hline Acuerdo & 43 & 29,5 & \\
\hline Total acuerdo & 4 & 2,7 & \\
\hline Número total & & & 147 \\
\hline
\end{tabular}

Como la tabla 1 refleja, el porcentaje mayor $(47,9)$ es el de informantes que no emplean los foros para recopilar información de carácter académico. El segundo porcentaje más alto es el de 29,5, que corresponde a los encuestados que por el contrario sí emplean los foros con fines de obtener información. A la vista de estos resultados, se podría decir que el empleo de foros para recopilar información académica ronda el 30\% de los encuestados, lo que hace pensar que aún no es un recurso muy establecido para este fin entre los estudiantes. Probablemente, en la medida en que se vaya usando más este recurso en las distintas asignaturas universitarias, los resultados podrían, sin duda, ir cambiando.

Tabla 2. Uso de blogs para recopilar información académica.

\begin{tabular}{lcll} 
& Frecuencia & Porcentaje & Total \\
\hline Total desacuerdo & 31 & 21,2 & \\
Desacuerdo & 53 & 36,3 & \\
Acuerdo & 57 & 39 & 147 \\
Total acuerdo & 5 & 3,4 & \\
Número total & & & \\
\hline
\end{tabular}

Como la tabla anterior refleja, el uso de blogs para recopilar información académica sí parece estar algo más extendido que el de foros, ya que 39\% de los encuestados afirma usar este recurso, frente a $36,3 \%$ que, por el contrario, no lo emplea. El blog es un recurso que, aunque aún no goza de una gran acogida por el alumnado universitario con fines académicos, sí que parece emplearse más que los foros. Tal vez, porque consideran este recurso más fiable en términos de la calidad que en estos se publica. 
Tabla 3. Uso de RSS para recopilar información académica

\begin{tabular}{lccc} 
& Frecuencia & Porcentaje & Total \\
\hline Total desacuerdo & 65 & 44,8 & \\
Desacuerdo & 60 & 41,4 & \\
Acuerdo & 18 & 12,4 & \\
Total acuerdo & 2 & 1,4 & 147 \\
Número total & & & \\
\hline
\end{tabular}

El uso de las redes sociales con el fin de recopilar información académica fue el tercer recurso por el que se preguntó y los resultados reflejan un extendido rechazo de este recurso como fuente de información académica con un $41,4 \%$ de estudiantes que se muestra en desacuerdo con el uso de las redes sociales, y un $44,8 \%$ que muestra un total desacuerdo. Esto implica que alrededor de un $85 \%$ de los encuestados no emplea las redes sociales con este fin. Tan sólo un $15 \%$ parece usar este recurso como fuente de información. Esto podría ser indicativo de que los generaciones jóvenes parecen asociar las redes sociales más al ocio y actividades de tiempo libre que al estudio y a lo académico.

A partir del análisis del uso de los foros, blogs y redes sociales para recopilar información de carácter académico, se podría decir que los blogs son los más extendidos entre los encuestados, seguido de los foros y, en último lugar, las redes sociales que apenas son empleadas por los informantes con este fin.

A continuación, nos centraremos en el estudio del siguiente bloque que giraba en torno al uso de recursos de la Web 2.0 para la gestión y reflexión de la información aprendida y estudiada.

\subsubsection{Bloque B: Gestionar y Reflexionar}

Tabla 4. Uso de blogs para elaborar textos reflexivos sobre lo que estudio o aprendo.

\begin{tabular}{lclc} 
& Frecuencia & Porcentaje & Total \\
\hline Total desacuerdo & 42 & 28,8 & \\
Desacuerdo & 61 & 41,8 & \\
Acuerdo & 39 & 26,7 & \\
Total acuerdo & 4 & 2,7 & 146 \\
Número total & & & \\
\hline
\end{tabular}

El primer recurso por el que se preguntó fue el blog con el fin de escribir en ellos textos reflexivos sobre lo que los informantes estudian o aprenden y los resultados muestran que el mayor porcentaje $(41,8)$ es el de encuestados que no emplean los blogs para reflexionar sobre lo aprendido. El segundo mayor porcentaje $(28,8)$ es el de estudiantes que tampoco usan este recurso con este fin. Por el contrario, un $26,7 \%$ y un $2,7 \%$ sí parece emplear los blogs para el estudio y la reflexión. Esto indica que este recurso aún no parece gozar de buena acogida entre los jóvenes estudiantes para afianzar lo aprendido con fines académicos. 
Tabla 5. Puesta en práctica de lo aprendido mediante reflexiones en blogs o foros.

\begin{tabular}{lclc} 
& Frecuencia & Porcentaje & Total \\
\hline Total desacuerdo & 42 & 28,6 & \\
Desacuerdo & 64 & 43,5 & \\
Acuerdo & 37 & 25,2 & \\
Total acuerdo & 4 & 2,7 & 147 \\
Número total & & & \\
\hline
\end{tabular}

Los resultados expuestos en la tabla anterior ponen de manifiesto que el porcentaje mayor $(43,5)$ es el de informantes que no usan los blogs o foros para poner en práctica lo que aprenden mediante reflexiones. No obstante, sí hay un porcentaje $(25,2)$ de estudiantes que sí emplean estos recursos para poner en práctica lo que aprenden que, aunque inferior, también parece considerable. Se puede vislumbrar una tendencia que podría ir en aumento en el futuro con respecto al empleo de estos recursos para la práctica de lo aprendido.

Tabla 6. Para elaborar trabajos en grupo con mis compañeros utilizo Wikis o Google Docs.

\begin{tabular}{lcll} 
& Frecuencia & Porcentaje & Total \\
\hline Total desacuerdo & 9 & 6,1 & \\
Desacuerdo & 28 & 19 & \\
Acuerdo & 67 & 45,6 & \\
Total acuerdo & 43 & 29,3 & 147 \\
Número total & & & \\
\hline
\end{tabular}

Con respecto al uso de Wikis o Google Docs para elaborar trabajos en grupo con compañeros, estos recursos parecen ser ampliamente utilizados por los encuestados. Un 45,6\% y un $29,3 \%$ de informantes confirma que emplea estos recursos en sus trabajos grupales. Esto implica que se trata de medios que están consolidados en lo que se refiere al uso con fines académicos y de aprendizaje.

El tercer bloque que se analizó fue el del uso de recursos de la Web 2.0 con la finalidad de practicar lo aprendido. 


\subsubsection{Bloque C: Practicar}

Tabla 7. Utilizar Podcasts para practicar de forma oral lo que aprendo.

\begin{tabular}{lclc} 
& Frecuencia & Porcentaje & Total \\
\hline Total desacuerdo & 27 & 18,5 & \\
Desacuerdo & 51 & 34,9 & \\
Acuerdo & 55 & 37,7 & \\
Total acuerdo & 13 & 8,9 & 146 \\
Número total & & & \\
\hline
\end{tabular}

El podcast fue el primer recurso por el que se indagó. Se preguntó a los informantes si ellos lo emplean para practicar actividades orales. Pensemos en aprendizaje de idiomas donde la parte oral tiene un gran peso. A esto respondieron los estudiantes que sí lo emplean un $37,7 \%$ y un 8,9 . Frente a estos, un $34,9 \%$ y un $18,5 \%$ de encuestados no lo emplea. En esto caso, se puede decir que los porcentajes rondan el $50 \%$ entre los que sí usan este medio y los que no, para practicar actividades orales. Estos resultados posiblemente cambien a favor de los que sí emplean los podcasts para hacer prácticas orales en el futuro en la medida en que este medio se vuelva más popular entre los estudiantes.

Tabla 8. Reflexionar en blogs o foros para poner en práctica lo que he aprendido.

\begin{tabular}{lclc} 
& Frecuencia & Porcentaje & Total \\
\hline Total desacuerdo & 20 & 13,6 & \\
Desacuerdo & 44 & 29,9 & \\
Acuerdo & 75 & 51 & 147 \\
Total acuerdo & 8 & 5,4 & \\
Número total & & & \\
\hline
\end{tabular}

El uso de blogs o foros como instrumentos para la reflexión y práctica de lo aprendido parece estar bastante extendido, ya que algo más de la mitad de los informantes (51\%) afirma emplear estos medios con estos fines. Los porcentajes de estudiantes que no emplean estos medios son algo inferiores $(29,9$ y 13,6$)$. Esto implica que se trata de recursos que progresivamente irán implantándose entre los jóvenes para ser usados con fines académicos.

Tabla 9. Practicar mediante la reflexión lo que he aprendido en redes sociales como Facebook, Tuenti o Twitter.

\begin{tabular}{lclc} 
& Frecuencia & Porcentaje & Total \\
\hline Total desacuerdo & 31 & 21,1 & \\
Desacuerdo & 56 & 38,1 & \\
Acuerdo & 44 & 29,9 & \\
Total acuerdo & 16 & 10,9 & 147 \\
Número total & & & \\
\hline
\end{tabular}


Dentro del bloque de práctica, la última pregunta que se formuló a los informantes fue si empleaban redes sociales como Facebook, Tuenti o Twitter para practicar lo aprendido a través de la reflexión. Los resultados demuestran que el mayor porcentaje de encuestados $(38,1$ y 21,1$)$ no emplea las redes sociales con este fin. Sin embargo, es necesario mencionar que alrededor de un $40 \%$ de los informantes $(29,9+10,9)$ sí afirma usar estos medios. Estos resultados se pueden traducir en que las redes sociales además de constituir un recurso fundamental para actividades de ocio entre los jóvenes, parece que se está también desarrollando en otras direcciones, como es el entorno académico y de estudios.

En la siguiente sección de este trabajo realizaremos un análisis comparativo entre los resultados obtenidos en las dos universidades y, por tanto, entre las dos titulaciones objeto de este estudio: País Vasco (titulación de Magisterio) y Las Palmas de Gran Canaria (titulación de Filología Inglesa y su actual equivalente grado en Lenguas Modernas)

\subsection{Comparativa entre ambas universidades y titulaciones}

Para establecer una comparativa entre los datos obtenidos en las dos universidades se emplearon tablas de contingencia dentro del programa SPSS. Los resultados obtenidos demuestran que los estudiantes de ambas universidades presentan un grado de conocimiento, manejo y familiaridad de los recursos por los que se preguntó bastante similar. Con el fin de codificar las respuestas obtenidas se estableció la misma escala de Likert de cuatro puntos como posibles respuestas.

Al igual que en el apartado anterior de este artículo el empleo de las distintas herramientas puede responder a distintos fines, por tanto, en el análisis que realizaremos distinguiremos los distintos bloques: A. búsqueda y recopilación de información, B. gestión y reflexión y C. práctica.

\subsubsection{Bloque A: Búsqueda y recopilación de información}

En lo que respecta al empleo de la herramienta foros con el fin de recopilar información, los resultados demuestran que existen diferencias entre las dos universidades objeto de este análisis, ya que en la Universidad del País Vasco, el mayor número de informantes (54) es el de aquellos que no emplean esta herramienta con estos fines, mientras que en la Universidad de Las Palmas de Gran Canaria son 35 los sujetos que parecen no usar los foros. Con respecto a las respuestas afirmativas que sostienen que sí usan los foros, el número es mayor en Las Palmas de Gran Canaria con 39 informantes, frente a los 18 del País Vasco. La tabla siguiente desglosa en detalle los resultados. 
Tabla 5. Uso de foros para recopilar información entre los informantes de ambas Universidades.

\begin{tabular}{llll} 
& País Vasco & Las Palmas GC & Total \\
\hline Total desacuerdo & 15 & 14 & \\
Desacuerdo & 40 & 30 & \\
Acuerdo & 15 & 28 & \\
Total acuerdo & 1 & 3 & 146 \\
Número total & & & \\
\hline
\end{tabular}

Los resultados demuestran que en ambas universidades son más los alumnos que no emplean los foros para recopilar información académica a través de este medio que los que sí lo hacen. En País Vasco $55(15+40)$ y en Las Palmas son $44(14+30)$ encuestados. Sin embargo, sí es cierto que hay cierta diferencia entre el número de alumnos que sí emplea este recurso con tales fines. En Las Palmas $31(28+3)$ encuestados afirma usar este medio, mientras que en País Vasco serían sólo $16(15+1)$ los que dicen usar este recurso. Asimismo, los alumnos de Filología Inglesa y grado en Lenguas Modernas usan más este recurso que los de Magisterio.

La siguiente herramienta por la que se preguntó fue el empleo de blogs. En este caso, los resultados afirman que nuevamente se trata de un recurso más usado por los sujetos de la Universidad de Las Palmas de Gran Canaria y, por tanto, los de Filología Inglesa y grado en Lenguas Modernas. En el caso de los alumnos que afirman no emplear los blogs, el mayor número está entre los informantes del País Vasco $(21+25)$, frente a $(10+28)$ estudiantes de Las Palmas. En cuanto a los alumnos que sí emplean los blogs, el mayor número es de Las Palmas y Filología Inglesa con $32+5$ informantes, frente a 25 alumnos de País Vasco y de Magisterio. Los resultados se reflejan en la siguiente tabla.

Tabla 6. Uso de blogs para recopilar información entre los informantes de ambas Universidades.

\begin{tabular}{llll} 
& País Vasco & Las Palmas GC & Total \\
\hline Total desacuerdo & 21 & 10 & \\
Desacuerdo & 25 & 28 & \\
Acuerdo & 25 & 32 & \\
Total acuerdo & 0 & 5 & 146 \\
Número total & & & \\
\hline
\end{tabular}

La tercera herramienta por la que se indagó con el objeto de completar la información que recibo en clase fue el uso de marcadores sociales (RSS). En este punto, los resultados son muy similares entre ambas universidades y titulaciones, ya que $36+26$ informantes del País Vasco y 29+34 encuestados de Las Palmas afirman no emplear este recurso. Muchos de ellos declararon, además, no conocer esta herramienta. De ahí que tan solo 9 informan- 
tes del País Vasco y $11(9+2)$ de Las Palmas de Gran Canaria empleen los RSS, como se refleja en la siguiente tabla.

Tabla 7. Uso de RSS para recopilar información entre los sujetos de ambas Universidades.

\begin{tabular}{llll}
\hline Total desacuerdo & 36 & 29 & \\
Desacuerdo & 26 & 34 & \\
Acuerdo & 9 & 9 & \\
Total acuerdo & 0 & 2 & 145 \\
Número total & & & \\
\hline
\end{tabular}

\subsubsection{Bloque B: Gestión y reflexión}

En esta segunda sección nos centraremos en el análisis del uso de recursos de la Web 2.0 con el objetivo de gestionar y reflexionar sobre la información. De ahí, que las cuestiones se encuentren bajo el encabezado de "en mi vida académica empleo los siguientes recursos".

Tabla 8. Reflexionar en Blogs o Foros para poner en práctica lo que he aprendido.

\begin{tabular}{llll} 
& País Vasco & Las Palmas GC & Total \\
\hline Total desacuerdo & 28 & 14 & \\
Desacuerdo & 30 & 34 & \\
Acuerdo & 14 & 23 & \\
Total acuerdo & 0 & 4 & 147 \\
Número total & & & \\
\hline
\end{tabular}

En lo que respecta al empleo de blogs o foros para poner en práctica lo aprendido, los datos reflejan que en el País Vasco (Magisterio) 58 estudiantes $(28+30)$ no emplean este recurso, mientras que en Las Palmas (Filología Inglesa / grado en Lenguas Modernas) son $48(14+34)$ los que tampoco usan estos medios. Si nos centramos en quienes sí usan estos recursos vemos que 14 alumnos en País Vasco y 27 estudiantes $(23+4)$ de Las Palmas sí emplean estos medios para la reflexión y práctica de los contenidos aprendidos. Significa esto que los informantes canarios parecen estar un tanto más familiarizados con el empleo de estos dos recursos con fines académicos. 
Tabla 9. Para elaborar trabajos en grupo con mis compañeros utilizo

Wikis o Google Docs.

\begin{tabular}{llll} 
& País Vasco & Las Palmas GC & Total \\
\hline Total desacuerdo & 7 & 2 & \\
Desacuerdo & 16 & 12 & \\
Acuerdo & 36 & 31 & \\
Total acuerdo & 13 & 30 & 147 \\
Número total & & & \\
\hline
\end{tabular}

En lo que respecta al empleo de Wikis o Google Docs para elaborar trabajos en grupo, observamos que se trata de una práctica bastante extendida. En este caso, el número de informantes que sí lo emplean es superior $(49=36+13)$ en País Vasco y en Las Palmas de GC $(61=31+30)$, aunque el número es algo mayor en el caso de Las Palmas. Los estudiantes que no emplean estos recursos son menos $(23=7+16)$ en País Vasco y en Las Palmas $(24=2+12)$. De nuevo, observamos que los encuestados canarios parecen tener mayor familiaridad con estos recursos con fines académicos que los vascos.

\subsubsection{Bloque C: Practicar}

Esta sección se centró en indagar por el uso de Podcasts para practicar la habilidad oral en las dos universidades.

Tabla 10. Utilizar Podcasts para practicar de forma oral lo que aprendo.

\begin{tabular}{llll} 
& País Vasco & Las Palmas GC & Total \\
\hline Total desacuerdo & 20 & 7 & \\
Desacuerdo & 27 & 24 & \\
Acuerdo & 24 & 31 & \\
Total acuerdo & 1 & 12 & 146 \\
Número total & & & \\
\hline
\end{tabular}

Como aparece recogido en la tabla anterior, los resultados reflejan que en País Vasco 47 informantes $(20+27=47)$ no emplean los podcasts con este objetivo, mientras que en Las Palmas son $31(7+24)$ los que no usan este recurso. Por el contrario, los que sí lo usan son $25(24+1)$ en País Vasco frente a $43(31+12)$ encuestados en Las Palmas que también lo emplean. En definitiva, observamos nuevamente que los informantes de Las Palmas tienden más a usar algunas herramientas de la Web 2.0 con fines académicos.

La siguiente pregunta se ocupó de averiguar si los sujetos utilizaban los blogs o foros para poner en práctica a través de la reflexión los contenidos aprendidos. 
Tabla 11. Reflexionar en Blogs o Foros para poner en práctica lo que he aprendido.

\begin{tabular}{llll} 
& País Vasco & Las Palmas GC & Total \\
\cline { 2 - 4 } Total desacuerdo & 15 & 5 & \\
Desacuerdo & 23 & 21 & \\
Acuerdo & 33 & 42 & \\
Total acuerdo & 1 & 7 & 147 \\
Número total & & & \\
\hline
\end{tabular}

Los resultados prueban que $38(15+23)$ alumnos de País Vasco o Magisterio frente a $26(5+21)$ de Las Palmas o Filología Inglesa no emplean estos recursos para practicar lo aprendido. Por el contario, 34 alumnos de País Vasco $(33+1)$ frente a 49 sujetos de Las Palmas (42+7) sí los emplean. Esto significa que es mayor el número de alumnos que sí emplean estos recursos que los que no lo hacen y que además en Las Palmas son más utilizados que en País Vasco.

El siguiente recurso por el que se preguntó fue el del empleo de redes sociales como Facebook, Tuenti o Twitter para practicar lo aprendido.

Tabla 12. Practicar mediante la reflexión lo que he aprendido en Redes Sociales tales como Facebook, Tuenti o Twitter.

\begin{tabular}{llll} 
& País Vasco & Las Palmas GC & Total \\
\hline Total desacuerdo & 21 & 10 & \\
Desacuerdo & 30 & 26 & \\
Acuerdo & 18 & 26 & \\
Total acuerdo & 3 & 13 & 147 \\
Número total & & & \\
\hline
\end{tabular}

Los resultados demuestran que 51 alumnos de País Vasco $(21+30)$ no emplean estos medios para practicar, mientras en Las Palmas el número de sujetos que no los usan es de $36(10+26)$. Asimismo, el número de estudiantes que sí usan las redes sociales para practicar es mayor en Las Palmas $(39=26+13)$ que en País Vasco $21(18+3)$. Una vez más observamos que los informantes de Las Palmas presentan mayor familiaridad con el empleo de recursos de la Web 2.0 con fines académicos.

\section{Conclusiones}

A partir del análisis realizado, hemos extraído las siguientes conclusiones:

En lo que respecta a la primera parte de la investigación donde se realiza un estudio general sobre el uso de algunos recursos de la Web 2.0 con fines académicos, concluiremos 
con que el empleo de estos medios parece gozar cada vez de más popularidad y aceptación por parte de los jóvenes. Si bien es cierto que algunos de ellos aún parecen no estar muy consolidados cuando se trata de utilizarlos con fines académicos, parece vislumbrarse una tendencia al alza en el empleo de éstos. Esto conecta directamente con la siguiente sección de este artículo.

En la segunda parte del estudio se llevó a cabo un análisis comparativo entre los estudiantes de las dos universidades objeto de este estudio. Los resultados reflejan que los informantes de Las Palmas parecen estar más familiarizados que los de País Vasco con el empleo de la Web 2.0 con fines académicos.

Resulta difícil encontrar una explicación para las diferencias que se han encontrado en este estudio entre ambas universidades y entre titulaciones. Sin embargo, con sus limitaciones, este trabajo prueba que la Web 2.0 parece empezar a convertirse en una herramienta útil para el mundo académico. Con el tiempo se irá, con toda probabilidad, imponiendo cada vez más en el mundo académico con todos los recursos que ofrece como medios para asimilar, afianzar y reflexionar sobre contenidos aprendidos.

En relación con el uso de las estrategias cognitivas por medio de las herramientas que Web 2.0 aporta, según los resultados obtenidos y siguiendo los bloques de estrategias que engloba, en general, la estrategia cognitiva y que se distinguen en la figura dos, señalamos lo siguiente:

1. Para la búsqueda y recopilación de la información, en general, la herramienta que los alumnos más utilizan son los blogs, seguidos de los foros y los RSS.

2. Cuando su objetivo es gestionar y reflexionar sobre lo aprendido optan, ante todo, por los wikis y Google Docs, dejando en un segundo plano instrumentos como los blogs y foros.

3. Para practicar, en cambio, en primer lugar eligen los blogs, después los podcasts y finalmente, las redes sociales.

A pesar de que, en general, los alumnos de la Universidad de las Palmas de Gran Canaria confiesan utilizar más internet para el desarrollo de las estrategias cognitivas, en lo que se refiere a la elección de los instrumentos que la Web 2.0 aporta, existe total coincidencia entre el alumnado de las dos universidades. Así, para la búsqueda y recopilación de la información, sobre todo, utilizan los blogs; para la gestión y la reflexión prefieren los wikis y el Google Docs; finalmente, para la práctica optan, en ambos casos, por los blogs y foros.

Por todo ello, consideramos que a pesar de que los alumnos parecen estar familiarizados con el uso de las herramientas Web 2.0, tienden a centrarse en algunas para el desarrollo de estrategias, tal y como podemos observar en los resultados expuestos. Es decir, el blog es la herramienta con mayor aceptación, lo que nos lleva a pensar que sería conveniente fomentar y trabajar con otras herramientas dentro de las asignaturas que cursan en sus carreras.

\section{Retos de Futuro}

El trabajo que se ha presentado constituye un estudio preliminar para seguir investigando en el futuro de forma más detallada y experimental. Por ello sería de gran interés llevar a cabo un estudio similar en el plazo de 5 a 10 años para determinar si los resultados obte- 
nidos varían o se mantienen parecidos a los de este trabajo. Nos aventuramos a pensar que con bastante probabilidad los resultados podrían cambiar considerablemente, debido a que el uso de recursos de la Web 2.0 está cada vez más presente no sólo en la vida cotidiana de las jóvenes y no tan jóvenes generaciones, sino que también son más empleados en los currículos de las distintas materias y asignaturas.

Por otro lado, también sería interesante establecer una comparativa entre estudiantes de dos países diferentes e incluso de dos titulaciones distintas: una técnica y una humanística, para establecer si existen diferencias entre los sujetos de ambas. Además de experimentar la aplicación de las estrategias planteadas mediante las herramientas web analizadas en contextos universitarios reales, es decir, dentro del proceso de enseñanza-aprendizaje en la universidad.

\section{Bibliografía}

Alonso F. (1993). Metacognición y Aprendizaje. Madrid: UCM.

Crespo, N. M. 2004. "La Metacognición: Las diferentes vertientes de una Teoría”, en Signos, 33, 48:97-115.

Cyr, P. (2000). Estrategiak bigarren hizkuntza baten irakaskuntzan (Traducción de: Boni Urkizu). Donostia: HABE (Itzulpen saila, 42).

Gallego J. (1997). Las estrategias cognitivas en el aula. Programas de intervención psicopedagógica. Madrid: Editorial Escuela Española.

O’Malley, M.; Chamot, A. (1990). Learning Strategies in Second Language Acquisition. Cambridge: Cambridge University Press.

Oxford, R. (1990). Language Learning Strategies: What Every Teacher Should know. Boston: Heinle \& Heinle Publishers.

Politzer, R. \& McGroarty, M. (1985). “An exploratory study of learning behaviors and their relationships to gains in linguistic and communicative competence.", in TESOL Quarterly, 19: 103-24.

Rubin, J. (1981). "Study of cognitive processes in second language learning”, Applied Linguistics, 11: $117-131$.

Skehan, P. (1997). Individual differences in second-language learning. London: Arnold.

Villanueva, M. L. (1997). "Estilos cognitivos y estilos de aprendizaje. Autonomía y aprendizaje de lenguas", en Becerra J. M. et al. (ed.), Los estilos de aprendizaje de lenguas. Granada: Grupo de Investigación de Lingüística Aplicada.

\section{Notas}

1. Quienes deseen conocer la encuesta que empleamos para la realización de este estudio, pueden obtenerla contactando con alguna de las autoras.

2. Asimismo, deseamos hacer constar que las doctoras Urtza Garay y Aintzane Etxebarría han elaborado las secciones $1,2,3,4,6,7$ y 8 de este artículo. La doctora Carmen Luján ha realizado las secciones 3, 4, 5, 6 y 7 del presente trabajo. 